\title{
Nucleotide excision repair proteins and PARP1/PAR interplay regulats protein assembly on damaged DNA
}

\author{
Nadejda Rechkunova \\ Lab of Bioorganic Chemistry \\ of Enzymes \\ ICBFM SB RAS \\ Novosibirsk, Russia \\ nadyarec@niboch.nsc.ru \\ Maria Sukhanova \\ Lab of Bioorganic Chemistry \\ of Enzymes \\ ICBFM SB RAS \\ Novosibirsk, Russia
}

\author{
Ekaterina Maltseva \\ Lab of Bioorganic Chemistry \\ of Enzymes \\ ICBFM SB RAS \\ Novosibirsk, Russia
}

\author{
Olga Lavrik \\ Lab of Bioorganic Chemistry \\ of Enzymes \\ ICBFM SB RAS \\ Novosibirsk, Russia
}

\author{
Yuliya Krasikova \\ Lab of Bioorganic Chemistry \\ of Enzymes \\ ICBFM SB RAS \\ Novosibirsk, Russia
}

\begin{abstract}
Nucleotide excision repair (NER) is one of the major mechanisms to prevent genomic DNA instability. This process removes a wide range of lesions distorting the double helix and bulky chemical adducts resulting from environmental factors or chemotherapeutic agents. The coordination of the assembly of the NER complexes and the sequential individual reactions is achieved through multiple DNA-protein and protein-protein interactions. The interactions of key protein factors of the NER process, xeroderma pigmentosum protein $C$ in the complex with RAD23B (XPC-RAD23B), xeroderma pigmentosum protein A (XPA), and replication protein A (RPA) with DNA mimicking NER intermediates and their modulation by the activity of poly(ADP-ribose)polymerase 1 (PARP1) and poly(ADP-ribose) have been analyzed. Using several biochemical approaches we have analyzed the influence of PARP1 and PAR synthesis on the interaction of the NER factors with damaged DNA. XPC-RAD23B, RPA and XPA are the targets of poly (ADP-ribosyl)ation catalyzed by PARP1, bind free poly(ADP-ribose) with an affinity depending on the PAR strand length and influence on PAR synthesis. Both XPC-RAD23B and XPA stimulate PAR synthesis in all experimental conditions whereas effect of RPA depends on the structure of DNA used for PARP1 activation. RPA inhibits PAR synthesis in the presence of ssDNA and stimulates it in the presence of DNA duplex, in particular containing a nick or a gap. RPA stimulation effect can be due to acceleration of the replacement of auto-(ADP-ribosyl)ated PARP1 in the complex with DNA by unmodified one. The data obtained suggest participation of RPA in regulation of PARP1 activity, which, in its turn, could play an important role in regulation of not only NER, but also replication-coupled repair and other DNA repair pathways. Our study clearly shows that PARP1 can be regarded as the universal regulator in DNA repair processes.
\end{abstract}

Keywords - nucleotide excision repair, poly(ADPribose)polymerase 1, DNA-protein interactions, protein-protein interactions

\section{Introduction}

Genetic stability of living organisms is substantially maintained by the action of the DNA repair systems. Regulation of the activity of the DNA repair systems is required to efficiently protect genomic DNA under genotoxic stress. One of the key mechanisms modulating the activity of DNA repair systems is the synthesis of poly(ADP-ribose) attached covalently to some repair proteins. Cellular DNA damage response by PAR synthesis is mainly mediated by PARP1, which is the most abundant and well-studied member of the PARP family $[1,2]$. Poly(ADP-ribosyl)ation targets are certain nuclear proteins including PARP1 itself. Poly(ADP-ribosyl)ation of the proteins can modulate their interactions with DNA due to the linkage to the negatively charged PAR [3] and can also be regarded as a signal of DNA damage. The PARylation reaction is reversible: PAR is cleaved by the poly(ADPribosyl)glycohydrolase (PARG) that additionally regulates the cellular level of protein poly(ADP-ribosyl)ation and PAR synthesis. Here we analyzed interactions of key protein factors of the NER process, xeroderma pigmentosum protein $\mathrm{C}$ in the complex with $\mathrm{RAD} 23 \mathrm{~B}$ (XPC-RAD23B), xeroderma pigmentosum protein $\mathrm{A}$ (XPA), and replication protein A (RPA) with DNA mimicking NER intermediates and their modulation by the activity of PARP1 and poly(ADP-ribose).

\section{Results and Discussion}

$P A R P 1$ modulates XPC-RAD23B interaction with DNA via poly(ADP-ribosyl)ation)

The XPC-RAD23B heterodimer is the major factor responsible for the primary damage recognition in DNA and initiating assembly of the NER complex. We found that electrophoretic mobility of XPC-RAD23B-DNA complex decreases in the condition of PAR synthesis and it is restored by the PARG treatment. It might correspond to formation of the covalent or noncovalent complexes of XPC-RAD23B with PAR [4].

Using ${ }^{32} \mathrm{P}$-labeled $\mathrm{NAD}^{+}$and immunoblotting analyses we have shown that both subunits of XPC-RAD23B heterodimer are PARylated by PARP1 in the presence of damaged DNA duplex. Direct interaction of these proteins was demonstrated in experiments with the fluorescence labeled PARP1. To demonstrate XPC-RAD23B PARylation relevance to the NER process we analyzed PARP1 activation by UV-induced DNA damages. We demonstrated that XPC-RAD23B is poly(ADP-ribosyl)ated by PARP1 in response to UV irradiation of plasmid DNA in dose dependent manner. These findings are in line with observations that UV irradiation triggers both stimulation of PAR synthesis and association of PARP1 with UV photolesions in chromatin [5]. Free or covalently bound to other proteins (including PARP1) PAR might serve as target for the attraction of XPC-RAD23B to lesions in DNA. PARP1 also influences XPA binding to DNA but in 
different manner. PARP1 displaces XPA from the complex with DNA and PAR synthesis inhibits XPA binding to DNA.

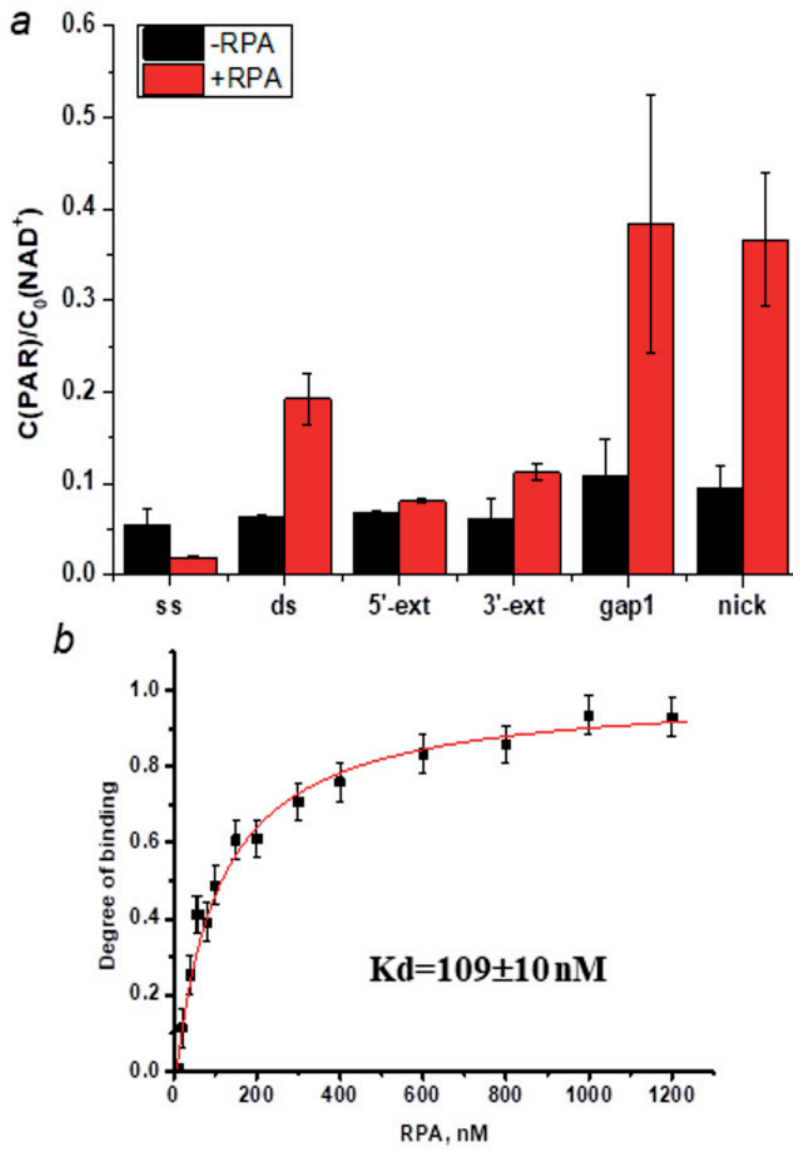

Fig. 1. RPA influences the PAR synthesis dependently on DNA structure (a) and physically interacts with PARP1.

To have non-visible rules on your frame, use the MSWord "Format" pull-down menu, select Text Box > Colors and Lines to choose No Fill and No Line.

\section{RPA modulates PARP1 activity in DNA dependent manner}

RPA is a major eukaryotic single-stranded DNA binding (SSB) protein, which is indispensable in DNA replication, repair, and recombination. RPA is heterotrimer consisting of p70, p32, and p14 subunits. We examined the efficiency of RPA PARylation by PARP1 in the presence of different DNA structures: ssDNA, double-stranded DNA (dsDNA), DNA duplexes with a 3'- or 5'-single-stranded extension (3'ext-DNA and 5'-ext-DNA, respectively), DNA duplex with a single-nucleotide gap (gap1-DNA), and DNA duplex with a single-strand break (nick-DNA).

For all DNA structures, the major reaction product corresponded to the product of PARP1 automodification. Modification of RPA, presumably the large p70 subunit, was also detected with the efficiency depended on the DNA structure. The maximal level of RPA modification was observed for the 3'-ext-DNA. For this structure, PARylation of $\mathrm{p} 32$ subunit was also displayed. In the case of ssDNA, efficiency of the proteins modification was decreased significantly in the presence of RPA.

RPA is not only a target PARylation but it also changes the efficiency of PARP1 automodification. Therefore, RPA can influence PARP1 activity. To check this possibility, we tested the effect of RPA on the PAR synthesis catalyzed by PARP1 in the presence of different DNA. RPA was shown to significantly increase the efficiency of PAR synthesis when the DNA structures with blunt ends were used. This effect was most visible with nick-DNA and gap1-DNA. For DNA structures with protruding single-stranded parts, RPA either had no significant effect (5'-ext-DNA, and 3 '-extDNA) or inhibited PAR synthesis (ssDNA) (Fig. $1 a$ ). RPAPARP1 interaction in the absence of DNA was detected by fluorescent titration (Fig. $1 \mathrm{~b}$ ).

RPA also influences leaving of PARP1 from DNA during PARylation. DNA-bound RPA can facilitate interaction of unmodified PARP1 with DNA. As a result, the replacement of modified PARP1 molecules by the unmodified ones on DNA accelerated, and the length of synthesized PAR was shortened [6]. The recent data on PAR synthesis at sites of DNA replication in normal $\mathrm{S}$ phase cells allowed indicating PARP1 is a sensor of unligated Okazaki fragments [7]. This observation is in line with our result that PARP1 interacts with RPA on nick/gap1-DNA.

\section{Conclusions}

The data obtained in this study clearly show that PARP1 interacts with and influences DNA binding of key NER factors which modulate its activity. Therefore, PARP1 can be regarded as the universal regulator in DNA repair processes.

\section{ACKNOWLEDGMENT}

The authors are grateful to Dr. Rashid Anarbaev for the contribution in preparation of the fluorescence labeled PARP1 and RPA and fluorescence anisotropy measurements.

\section{REFERENCES}

[1] N. Ogata, K. Ueda, M. Kawaichi, and O.Hayaishi, "Poly(ADPribose) synthetase, a main acceptor of poly(ADP-ribose) in isolated nuclei”, J. Biol. Chem., vol. 256, pp. 4135-4137, May 1981.

[2] D. D'Amours, S. Desnoyers, I. D'Silva, and G.G. Poirier, "Poly(ADP-ribosyl)ation reactions in the regulation of nuclear functions", Biochem. J., vol. 342, pp. 249-268, September 1999.

[3] J.M. de Murcia, C. Niedergang, C. Trucco, M. Ricoul, B. Dutrillaux, M. Mark, F. J. Oliver, M. Masson, A. Dierich, M. LeMeur, C. Walztinger, P. Chambon, and G. de Murcia, "Requirement of poly(ADP-ribose) polymerase in recovery from DNA damage in mice and in cells", Proc. Natl. Acad. Sci. U S A, vol. 94, pp. 73037307, July 1997.

[4] E.A. Maltseva, N.I. Rechkunova, M.V. Sukhanova, and O.I. Lavrik, "Poly(ADP-ribose) Polymerase 1 Modulates Interaction of the Nucleotide Excision Repair Factor XPC-RAD23B with DNA via Poly(ADP-ribosyl)ation", J. Biol. Chem., vol. 290, pp. 2181121820, September 2015.

[5] M.D. Vodenicharov, M.M. Ghodgaonkar, S.S. Halappanavar, R.G. Shah, and G.M. Shah, "Mechanism of early biphasic activation of poly(ADP-ribose) polymerase-1 in response to ultraviolet B radiation” J. Cell. Sci., vol. 118, pp. 589-599, February 2005.

[6] E.A. Maltseva, Y.S. Krasikova, M.V. Sukhanova, N.I. Rechkunova, and O.I. Lavrik, "Replication protein A as a modulator of the poly(ADP-ribose)polymerase 1 activity", DNA Repair, vol. 72, pp. 28-38, December 2018.

[7] H. Hanzlikova, I. Kalasova, A.A. Demin, L.E. Pennicott, Z. Cihlarova, K.W. Caldecott. "The importance of poly(ADP-ribose) polymerase as a sensor of unligated Okazaki fragments during DNA replication”, Mol. Cell., vol. 71, pp. 319-331, 2018. 